panzee-human alignments and the time of divergence, the estimated rate of formation of processed pseudogenes is 40-60 per Mya (D. Torrents, personal communication, for the Chimpanzee Genome Sequencing Consortium). Most such processed pseudogenes are 'dead on arrival', however, because of copying errors that occur during their creation. Even those that initially contain an intact open reading frame are not typically expressed, because they lack promoters. It is reasonable to speculate that GLUD2 happened by chance to insert adjacent to brain-specific promoter element(s), which then allowed selective pressure to support and enhance its continued expression. Partial deficiency of glutamate dehydrogenase activity has been found in individuals with various neurodegenerative disorders ${ }^{11}$, and excitotoxic neuronal death can be caused by accumulation of excess glutamate ${ }^{14}$. Given that GLUD2 is an ape-specific gene, it is difficult to test its importance in the standard rodent models. As it is located on the X chromosome, however, there is a reasonable likelihood that somewhere in the human population are males who carry GLUD2 mutations. Finding such individuals and studying their phenotypes would be a large step towards understanding the importance of GLUD2 in human brain evolution and function.

Thus, in addition to losing their tails, our ape ancestors seem to have been selected for multiple genetic modifications that favored the emergence of their specialized brains. The current work ${ }^{10}$ provides yet another piece to this part of the complex puzzle of human origins. In the final analysis, genes alone cannot explain the human brain. We must remember that the ape brain in general and the human brain in particular owe many of their sophisticated abilities to a intimate synergy between nature (genes) and nurture (environment). Thus, even if we eventually find all the needles in this haystack, the human mind will ultimately be explained only as "Nature via Nurture"15.
1. Gagneux, P. \& Varki, A. Mol. Phylogenet. Evol. 18, 2-13 (2001).

2. Olson, M.V. \& Varki, A. Nat. Rev. Genet. 4, 20-28 (2003).

3. Watanabe, H. et al. Nature 429, 382-388 (2004).

4. Olson, M.V. \& Varki, A. Science 305, 191-192 (2004).

5. Grossman, L.I., Wildman, D.E., Schmidt, T.R. \& Goodman, M. Trends Genet. (in the press).

6. Zhang, J. Genetics 165, 2063-2070 (2003).

7. Evans, P.D. et al. Hum. Mol. Genet. 13, 489-494 (2004).

8. Kouprina, N. et al. PLoS Biol. 2, E126 (2004).

9. Evans, P.D., Anderson, J.R., Vallender, E.J., Choi, S.S. \& Lahn, B.T. Hum. Mol. Genet. 13, 1139-1145 (2004).

10. Burki, F. \& Kaessmann, H. Nat. Genet. 36, 1061-1063 (2004).

11. Shashidharan, P. et al. J. Biol. Chem. 269, 16971-16976 (1994).

12. Balakirev, E.S. \& Ayala, F.J. Annu. Rev. Genet. 37, 123-151 (2003).

13. Torrents, D., Suyama, M., Zdobnov, E. \& Bork, P. Genome Res. 13, 2559-2567 (2003).

14. Sattler, R. \& Tymianski, M. Mol. Neurobiol. 24, 107-129 (2001).

15. Ridley, M. Nature Via Nurture: Genes, Experience, and What Makes Us Human (HarperCollins, New York, 2003).

\title{
Shifting insulator boundaries
}

\author{
Sangkyun Jeong \& Karl Pfeifer
}

\begin{abstract}
A new study shows that poly(ADP-ribosyl)ation of CTCF regulates its activity as a transcriptional insulator without altering its DNA binding properties. This mechanism provides cells with the flexibility to regulate gene expression without altering fundamental epigenetic programs.
\end{abstract}

Transcriptional insulators are DNA elements that set boundaries on the actions of enhancer and silencer elements and thereby organize the eukaryotic genome into regulatory domains ${ }^{1}$. All vertebrate insulators characterized to date use the versatile CTCF protein. CTCF uses various combinations of its 11 zinc fingers to recognize a variety of unrelated DNA sequences $^{2}$. Once bound to DNA, CTCF can function as a transcriptional insulator, repressor or activator depending on the context of the binding site ${ }^{3}$. CTCF also seems to determine gene expression patterns indirectly by establishing or maintaining specific epigenetic programs in the

\footnotetext{
Sangkyun Jeong and Karl Pfeifer are in the Laboratory of Mammalian Genes and Development, Building 6B-206, National Institute of Child Health and Human Development, National Institutes of Health, Bethesda, Maryland 20892, USA. e-mail:pfeiferk@mail.nih.gov
}

cell $^{3,4}$. On page 1105 of this issue, Yu et al. ${ }^{5}$ identify a post-translational mechanism for regulating CTCF insulator activity that adds to its versatility and its ability to effectively manage epigenetic programs.

Yu et $a l .{ }^{5}$ examine the action of CTCF using the imprinted Igf2-H19 locus as their model system. Figure 1a depicts the organization of the locus, including shared downstream enhancer elements and the H19 imprinting control region (ICR $)^{6}$. Binding of CTCF to its four recognition sites in the ICR prevents enhancer activation of the distal Igf2 promoter but not the proximal H19 promoter, demonstrating the ability of CTCF to act as classical transcriptional insulator. On the paternal chromosome, methylation of the ICR DNA sequences prevents CTCF binding and, therefore, Igf2 is expressed. The demonstration that regulation of CTCF binding could modulate transcriptional insulation was a key finding in both the imprinting and insulator fields.

\section{Let's PARley}

By re-examining the unmethylated (maternal) ICR, Yu et al. ${ }^{5}$ document binding of a poly(ADP-ribosyl)ated protein on this element. They then provide circumstantial but compelling evidence that the poly(ADP-ribosyl)ated protein is CTCF. Poly(ADPribosyl)ation is a protein modification in which homopolymers of up to 200 ADPribose units are attached by the action of poly(ADP-ribose) polymerases (PARPs) ${ }^{7,8}$. As each ADP-ribose has a negative charge, poly(ADP-ribosyl)ation causes a substantial change in the three-dimensional and electrostatic properties of the target protein.

Most notably, Yu et al. ${ }^{5}$ demonstrate that insulator function, but not CTCF binding, is sensitive to the PARP inhibition. That is, blocking PARP activity results in reactivation of the maternal Igf2 gene even while CTCF remains bound to the ICR (Fig. 1b). Thus, this study uncovers a unique mechanism for regulating insulator activity without disrupting CTCF-DNA interactions. A brilliant feature of 
this report is the demonstration that this mechanism is not restricted to the unusual case of the imprinted Igf2 locus. Rather, the link between CTCF and poly(ADP-ribosyl)ation, and between poly(ADP-ribosyl)ation and insulation, extends to more than 100 mouse target sites.

These new results contradict, in some sense, the chief paradigm for PARP regulation of DNA metabolism ${ }^{8}$. The best characterized role for any PARP enzyme is the role of PARP1 in mediating DNA repair. PARP1 is a DNA binding protein whose poly(ADP-ribosyl)ation activity is increased up to 500 -fold by singlestrand breaks in DNA. Activated PARP induces poly(ADP-ribosyl)ation of proximal proteins, including itself, which in turn promotes their removal from the DNA. A similar mechanism of chromatin clearing is postulated to explain the role of PARP in transcriptional regulation in Drosophila melanogaster ${ }^{9}$. Poly(ADP-ribosyl)ation is transient both because poly(ADPribosyl)ated PARP cannot bind DNA and because long ADP-ribose polymers activate a hydrolase (PARG) that catalyzes polymer removal. In contrast, at the CTCF insulators, poly(ADP-ribosyl)ation is the default state. Notably, poly(ADP-ribosyl)ation does not alter the ability of CTCF to bind to DNA. Rather, the mechanisms described by $\mathrm{Yu}$ et al. present a means to activate and deactivate insulators without removing CTCF from the DNA.

\section{Remembering the past}

The ability to regulate CTCF function without altering its DNA binding may be crucial, given its multiple roles in the cell. CTCF not only regulates gene expression as a classical transcription factor but also seems to have a crucial role in establishing or maintaining epigenetic programs ${ }^{3,4}$. These roles are well documented at the Igf2 locus. Preventing binding of CTCF to the maternal ICR (by cis or trans mutations) not only results in loss of Igf2 insulation but also leads to the inappropriate methylation of the entire ICR and the neighboring $H 19$ promoter (Fig. 1c $)^{10}$. In other words, a temporary loss of CTCF binding can cause a permanent change in the epigenetic identity of the maternal chromosome.

Similarly, CTCF might have a crucial role in determining the epigenetic state of other loci, not just imprinted ones. In particular, maintenance of gene expression patterns requires that the cell keep track of a variety of developmental decisions, even in the absence of the original environmental and developmental cues that induced these decisions. Insofar as CTCF has a role in epigenetic programming, regulation of its activity without removing it

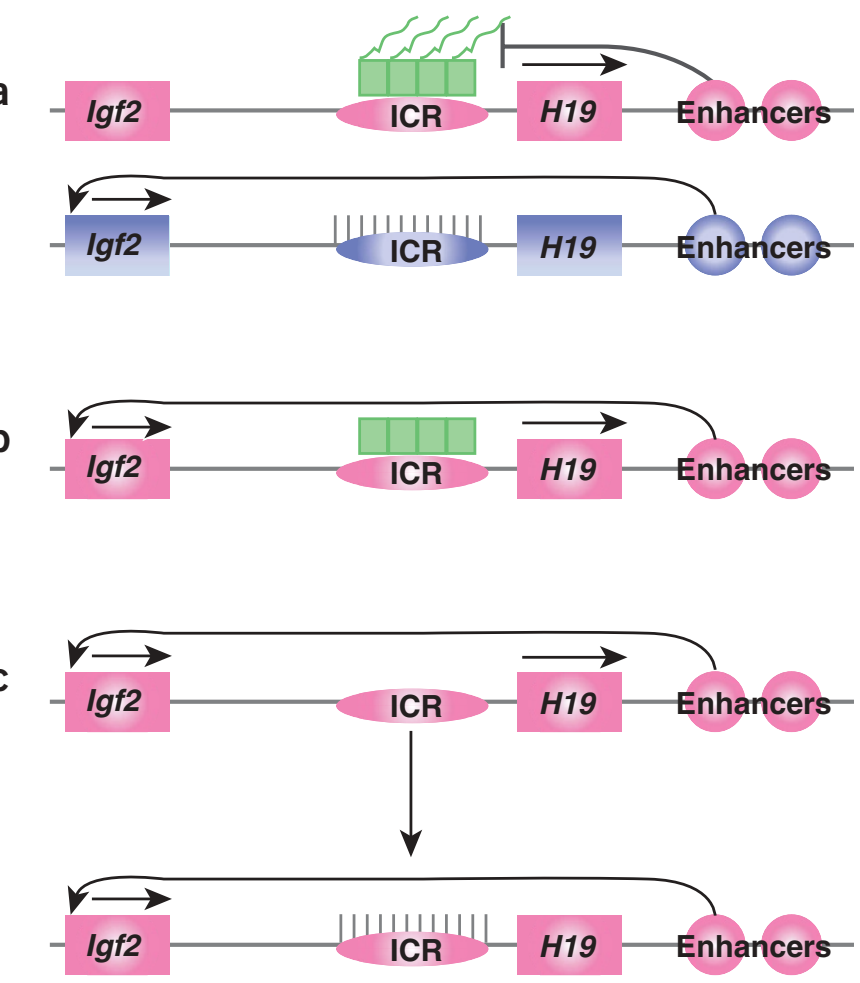

Figure 1 Regulating the insulator activity of the H19 ICR. (a) Maternal (pink) and paternal (blue) copies of the Igf2-H19 locus. Cytosine methylation (vertical lines) blocks CTCF (green boxes) binding. (b) Poly(ADPribosyl)ation (squiggled lines) is essential for CTCF insulator activity but not for DNA binding. (c) Any protein modification that disrupts CTCF binding will also disrupt the epigenetic program by leading to methylation of the maternal ICR. Thus, reactivation of Igf2 is irreversible.

from the DNA provides a mechanism whereby the cell maintains flexibility in gene expression without losing information about its developmental past.

Although there is no essential need for reactivation of maternal $I g f 2$ in mammals, there are medical problems associated with inappropriate expression of this allele ${ }^{11}$. Therefore, another contribution of this paper is the demonstration of a new mechanism for loss of imprinting at Igf2, in addition to the established genetic (mutation of CTCF binding sites ${ }^{12}$ or of $\mathrm{CTCF}^{13}$ ) and epigenetic (inappropriate DNA methylation of the ICR) mechanisms. In particular, the mechanisms described by Yu et al. might account for mysterious cases of biallelic expression of $I g f 2$ in which the maternal allele shows normal methylation.

The PARP enzymes (up to 18 in mammals ${ }^{14}$ ) and specific mechanisms that regulate and target CTCF poly(ADP-ribosyl)ation remain to be determined. As the number of PARPs and the known roles for poly(ADPribosyl)ation steadily expand, searches for new and specific roles for PARP proteins and for specific inhibitors of PARP enzyme activity intensify. Future studies to identify the specific PARP proteins responsible for CTCF modification, the protein partners and mechanism that target the enzyme to DNA-bound CTCF and the effects of enzyme-specific inhibitors will provide further mechanistic insights and may suggest possible therapeutic applications.

1. Kuhn, E.J. \& Geyer, P. Curr. Opin. Cell Biol. 15, 259-265 (2003).

2. Flippova, G.N. et al. Mol. Cell. Biol. 16, 2802-2813 (1996).

3. Ohlsson, R., Renkawitz, R. \& Lobanenkov, V. Trends Genet. 17, 520-527 (2001).

4. Klenova, E.M., Morse, H., Ohlsson, R. \& Lobanenkov, V.V. Semin. Cancer Biol. 12, 399-414 (2002).

5. Yu, W. et al. Nat. Genet. 36, 1105-1110 (2004).

6. Verona, R.I., Mann, M.R. \& Bartolomei, M.S. Annu. Rev. Cell Dev. Biol. 19, 237-259 (2003).

7. Smith, S. Trends Biochem. Sci. 26, 174-179 (2001).

8. Ziegler, M. \& Oei, S.L. Bioessays 23, 543-548 (2001).

9. Tulin, A. \& Spradling, A.C. Science 299, 560-562 (2003).

10. Lewis, A. \& Murrell, A. Curr. Biol. 14, R284-R286 (2004).

11. Reik, W. et al. Int. J. Dev. Biol. 44, 145-150 (2000).

12. Sparago, A. et al. Nat. Genet. 36, 958-960 (2004).

13. Filippova, G. et al. Cancer Res. 62, 48-52 (2002).

14. Ame, J.-C., Speniehauer, C. \& de Murcia, G. Bioessays 26, 882-893 (2004). 